\title{
108
}

\section{Selected Disorders of the Female Reproductive System}

\author{
Gary R. Newkirk and Patricia Ann Boken
}

Pelvic pain is one of the most vexing problems encountered by the family physician. The monetary and psychosocial costs are unknown but are assumed to be high. In the United States about $40 \%$ of all laparoscopies are done for the evaluation and treatment of pelvic pain. ${ }^{1}$

\section{Evaluation of Pelvic Pain}

\section{Clinical Presentation}

Patients with pelvic pain present with a wide variety of complaints and physical findings. To better understand the causes of pelvic pain and assist with the initial evaluation, it is helpful to distinguish acute pelvic pain from chronic pelvic pain. Chronic pelvic pain is present for 6 months or more and acute pelvic pain for less than 6 months. Chronic pelvic pain syndrome is characterized by incomplete response to treatment, impairment at work or home, signs of depression, and pain that is worse than would be expected on the basis of the pathologic process ${ }^{2}$ (see Chapter 61).

\section{Diagnosis}

Effective evaluation of pelvic pain begins with a search for organic causes that require immediate therapy. An integrative approach that relates psychological, historical, and physical factors from the outset is essential. When taking the history it is helpful to differentiate acute from chronic pelvic pain because this distinction refines the differential diagnoses (Tables 108.1 and 108.2). A detailed history is obtained clarifying the location, severity, and inciting and relieving factors of the pain. Careful menstrual, pregnancy, and sexual histories are sought. Contraceptive use, previous pelvic problems or surgery, and previous diagnostic and therapeutic efforts are clarified. The psychosocial history includes clarification of prior sexual abuse, personal relationships, and exploration of the patient's efforts to cope with, and her fears about, the cause of pain.

Research has shown a high incidence of sexual abuse associated with chronic pain. ${ }^{3}$ Furthermore, current psychiatric diagnoses, especially depression, and substance abuse correlate with pelvic pain. Rates of childhood or adult sexual or physical abuse are as high as $48 \%$ in patients presenting with chronic pelvic pain. ${ }^{3}$

Formal psychological consultation may be useful for assessing the psychiatric contribution to chronic pelvic pain. In most instances a psychiatric diagnosis modifies the patient's perception and significance of pelvic pain and rarely is the sole cause of symptoms.

\section{Physical Examination}

The physical examination includes careful abdominal, musculoskeletal, and pelvic examination. In addition to the routine abdominal examination, the examiner looks for trigger points in the abdominal wall. The abdominal wall has been identified as a common source of pain for both acute and chronic forms of pelvic pain. ${ }^{4}$ The abdominal wall is palpated with the patient's abdominal muscles stretched (supine) and relaxed (knees bent), so trigger points typical of the myofascial syndrome can be detected. ${ }^{2}$ During the pelvic examination the external genitalia are examined for signs of sexually transmitted diseases or trauma. The bimanual examination evaluates the introitus, levator sling, vaginal walls, cervix, uterus, adnexa, septum, and cul-de-sac. The urethra is palpated from within the vagina, as significant pain in this area implies the urethral syndrome related to chlamydial infection. The patient is requested to contract and relax the vagina around the examining fingers to assist in the detection of vaginismus. An attempt is made to re-create the pain on the bimanual examination including palpation and movement of the cervix. If pressing on the posterior fourchette or bottom 
Table 108.1. Causes of Acute Pelvic Pain and Initial Management

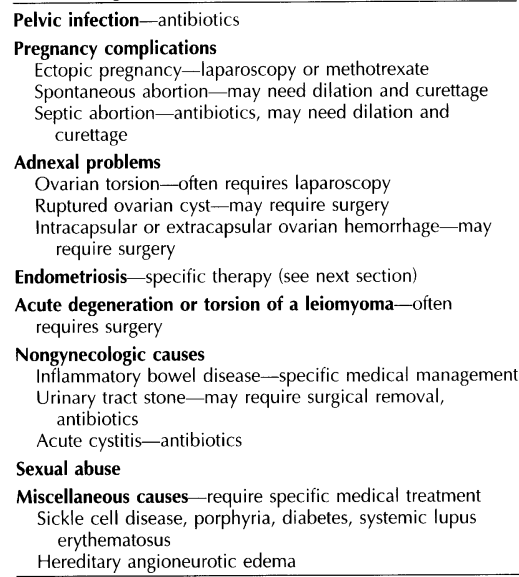

of the vagina reproduces the pain, there is a high likelihood that the pain has a significant psychological or musculoskeletal origin. Pain of pelvic organ origin may be noted ventrally or both dorsally and ventrally but almost never presents as dorsal back pain alone. The cul-de-sac is palpated for abnormalities such as nodules, which can be found with endometriosis. In most instances the cervix is screened or cultured for gonorrhea and Chlamydia.

\section{Table 108.2. Causes of Chronic and Cyclic Pelvic Pain}

Chronic pelvic pain
Endometriosis
Chronic salpingitis
Severe pelvic adhesions
Adenomyosis
Pelvic congestion syndrome
Nongynecologic causes
Musculoskeletal
Inflammatory bowel disease
Irritable bowel syndrome
Diverticulitis
Interstitial cystitis
Referred pain
Psychological
Cyclic pelvic pain
Mittelschmerz
Primary dysmenorrhea
Secondary dysmenorrhea
Adenomyosis
Endometrial polyp
Pedunculated submucous myoma
Premenstrual tension syndrome

Musculoskeletal dysfunction often contributes to the signs and symptoms of chronic pelvic pain and in many cases is the primary factor. ${ }^{5}$ King et $\mathrm{al}^{6}$ found that a typical pattern of faulty posture, termed typical pelvic pain (TPP) posture, was found in $75 \%$ of patients studied with chronic pelvic pain. The posture consists of exaggerated lordotic posture of the lumbar spine, anterior tilt of the pelvis, and kyphosis of the thoracic spine. Referred sources of pain must also be considered. ${ }^{6,7}$

\section{Diagnostic Studies}

The initial laboratory evaluation for pelvic pain includes a pregnancy test if the patient is of reproductive age, complete blood count (CBC), urinalysis, and erythrocyte sedimentation rate (ESR). Vaginal probe ultrasonography can confirm intrauterine pregnancies as early as 4 to 5 weeks and can assist in the evaluation of women with suspected ectopic pregnancy. The presence of fluid in the posterior cul-de-sac seen on the ultrasound scan suggests a pathologic process, but this finding has poor specificity. Ultrasonography may also be useful for patients who are difficult to examine because of obesity, are unable to relax, or whose pain prevents palpation during the bimanual examination. Unfortunately, abdominal ultrasonography is sometimes overutilized, as the likelihood of positive findings in a patient with completely normal pelvic examination is low and may contribute to further unnecessary imaging and heightened physician and patient anxiety. For example, ovarian cysts are a common finding on ultrasound scans and may be up to $5 \mathrm{~cm}$ in diameter; yet they are not the source of pain unless careful bimanual palpation of these cysts duplicates the pain.

Diagnostic laparoscopy remains an important tool for evaluating pelvic pain. ${ }^{8}$ Laparoscopy is the gold standard for diagnosing such conditions as pelvic inflammatory disease (PID), ectopic pregnancy, and ovarian torsion. The cause of chronic pelvic pain in patients under age 30 is most often endometriosis or chronic PID. Older patients are most likely to have adenomyosis, leiomyomas, endometriosis, or symptoms due to pelvic relaxation. Pelvic adhesions are the only finding in $10 \%$ to $25 \%$ of laparoscopies for chronic pelvic pain, but studies are still not clear whether adhesions are incidental findings or are the cause of the pain. Another benefit of diagnostic laparoscopy is to offer the patient reassurance. In one study that followed patients with chronic pelvic pain after laparoscopy had excluded pelvic pathology, $22 \%$ were pain-free at their 6 week follow-up appointment, and $40 \%$ reported decreased pain. ${ }^{9}$

Other diagnostic studies that may aid in the diagnosis include urine cultures, barium enema, intravenous pyelography, and empiric nerve blocks to help confirm the origin of pain (e.g., symptomatic adenomyosis).

\section{Management}

The patient with acute, severe pelvic pain and deteriorating clinical condition often requires abdominal exploration. In most other instances, a careful history and physical examination indicate the most likely nonsurgical causes of acute pelvic pain. Table 108.1 outlines initial management steps for the various causes of acute pelvic pain. 
Narcotics can be used with acute pelvic pain on a selective basis, when the diagnosis is assured, and for a short time. Narcotics should not be used for chronic pelvic pain. Nonsteroidal antiinflammatory drugs (NSAIDs) are the best choice for long-term causes of pelvic pain. Antidepressants help with the management of chronic pelvic pain regardless of whether symptoms of classic depression are identified, and they can be tried on an empiric basis. Low-dose amitriptyline $(25 \mathrm{mg})$ was significantly more effective than placebo in reducing such pain. ${ }^{10}$

Hormone treatment with oral contraceptives, Depo-Provera, and gonadotropin-releasing hormone $(\mathrm{GnRH})$ analogues may be helpful in suppressing ovarian function to treat conditions such as endometriosis, adenomyosis, fibroids, dysmenorrhea and ovarian cysts. Definitive treatment with antibiotics and the occasional need to drain pelvic abscesses surgically are the main strategies for managing PID.

Several studies have reported the importance of a multidisciplinary management for treatment of chronic pelvic pain (CPP). Sexual and marital counseling, family therapy, and behavior modification therapy are interventions to be considered. Regular office visits should be scheduled to decrease the patient's need to justify visits by experiencing more pain The frequency of these visits can be gradually tapered as the patient improves. Realistic goals must be established early regarding the management of CPP. ${ }^{11}$ Adapting and coping with CPP is considered the therapeutic goal, with complete resolution of symptoms unlikely.

Adjunctive treatment includes nerve blocks and nerve interruption surgery. Paracervical blocks may have a diagnostic and therapeutic value, as a response to this trial of analgesia strongly implies a uterine source for the pelvic pain Fascial injections were advocated by Ling and Slocumb $b^{4}$ for myofascial origins of pelvic pain. Laser uterine nerve ablation therapy has had mixed results when used to manage uterine sources of pain. Patients often adamantly demand a hys terectomy, especially when the search for the etiology of CPP becomes nonproductive. Interestingly, in one study looking at the outcome of women undergoing hysterectomy with CPP $78 \%$ showed significant improvement. ${ }^{12}$

Other, less traditional therapies for CPP include physical therapy for musculoskeletal dysfunction, stressing management through lifelong attention to posture, strength, and flexibility; transcutaneous electrical nerve stimulation (TENS) for musculoskeletal abdominal wall or back pain; acupuncture; and relaxation and biofeedback.

\section{Prevention}

Early management of the symptoms of acute pelvic pain and identifying the source decreases the possibility of symptoms becoming chronic. In the patient who is sexually active, counseling regarding sexually transmitted diseases (STDs) and their prevention is always prudent. The use of estrogen in postmenopausal women may be helpful for preventing pelvic pain due to vaginismus. The use of NSAIDs for 3 days prior to menstruation and during menses is beneficial in preventing pelvic pain caused by dysmenorrhea. A thorough history early in the investigation of CPP sufferers, such as clarifying sexual or physical abuse, may avoid extensive medical evaluations and unnecessary surgical procedures (see Chapter 27). Appropriate posture along with good physical conditioning, nutrition, and regular exercise may prevent the musculoskeletal origins of pelvic pain.

\section{Family and Community Issues}

The multiple causes of pelvic pain make its treatment and diagnoses challenging. Family physicians are uniquely qualified to care for patients with pelvic pain by virtue of their psychosocial evaluation skills, long-term management potential, and general ability to coordinate care from various consultants. Education of the community about sexual abuse, STDs, PID, endometriosis, depression, and other factors associated with pelvic pain can be offered by the family physician.

\section{Endometriosis: Diagnosis and Therapy}

The presence of tissue that is biologically and morphologically similar to normal endometrium in locations beyond the endometrial cavity is termed endometriosis. ${ }^{13}$ Endometriosis affects approximately $7 \%$ to $10 \%$ of premenopausal women. ${ }^{14}$ The prevalence in the general population may be underestimated, as not all endometrial lesions exhibit classic morphologic findings at laparoscopy. ${ }^{15}$ Endometriosis is found in $30 \%$ to $60 \%$ of women who present for infertility evaluation. ${ }^{16}$ Sixty to seventy percent of women with endometriosis are nulliparous, and most have a family history. ${ }^{17}$ The pathophysiology of endometriosis remains incompletely characterized and poorly understood. The most widely accepted explanation for endometriosis focuses on the concept of retrograde flow of menstrual fluid back through the fallopian tubes with implantation of viable endometrial tissue in the free pelvis. ${ }^{18}$ This explanation contrasts with the coelomic metaplasia theory, which argues that undifferentiated coelomic epithelial cells remain dormant on the peritoneal surface until the ovaries produce hormones sufficient to stimulate this ectopic tissue. ${ }^{14}$ Despite the controversies surrounding pathophysiology, endometriosis remains one of the most common gynecologic diseases in women during their reproductive years.

\section{Clinical Presentation}

Endometriosis is rarely life-threatening but frequently becomes life-altering. Patients with endometriosis present a wide range of clinical symptoms, and frequently there is poor correlation between these symptoms and the extent of endometrial implants within the pelvis. Dysmenorrhea (50\%), infertility $(25-50 \%)$, pelvic pain and dyspareunia $(20 \%)$, and menstrual irregularities (12-14\%) are the most common complaints. ${ }^{19}$ Pain can be diffuse or localized to the organs involved. Other, less common symptoms include low back pain, dysuria, hematuria, and diarrhea, which classically occur before or during menses. Despite classic descriptions of the disease, endometriosis may present with some, none, or all of these symptoms, which may or may not correlate with the men- 
strual cycle. Textbooks describe the typical endometriosis patient as in her late twenties or early thirties, Caucasian, and frequently nulliparous. In reality, endometriosis occurs with all races from early adolescence to the perimenopausal period.

Endometriosis should also be considered in women initially presenting for evaluation of infertility. ${ }^{20}$ How endometriosis contributes to infertility is not known, and likely more than one mechanism is involved. Explanations include altered anatomy, ovulatory dysfunction, hormonal abnormalities, autoimmunity, toxic pelvic factors, altered sexual functioning, and increased spontaneous abortions. ${ }^{16,19}$ Even though it appears that the number of pelvic implants does not correlate well with the severity of dysmenorrhea and dyspareunia, the probability of successful conception appears to be inversely related to the severity of the disease. ${ }^{21}$ Recent prospective controlled trials suggest that minimal to mild endometriosis is not associated with reduced fecundity. ${ }^{22}$

\section{Diagnosis}

Patients presenting with pelvic pain, dyspareunia, dysmenorrhea, abnormal bleeding, or infertility should prompt the clinician to consider endometriosis as a diagnosis. Physical findings include uterosacral nodularity, retroversion of the uterus, limited pelvic mobility, adnexal masses, and diffuse or focal tenderness. Unfortunately, neither the history nor the physical examination confirms the diagnosis of endometriosis. The diagnosis of endometriosis requires direct visual and histologic confirmation obtained during laparoscopy or laparotomy. A uniform system of classification based on the presence, location, and quality of adhesions, endometriomas, and tubal distortion has been formulated by the American Fertility Society (AFS). The AFS classifies endometriosis as minimal, mild, moderate, and severe (stages I to IV, respectively). ${ }^{23}$ During laparoscopy or laparotomy, endometriosis may appear as classic brown lesions that may be focal (e.g., ovarian) or diffuse (e.g., peritoneal). Reddish blue nodules on the peritoneum, uterine ligaments, or pelvic viscera are a common finding as well. Focal scarring or retraction of the peritoneum, diffuse adhesions, and distortion of uterine, ovarian, and tubal anatomy can be seen. Ovarian implants may result in the formation of an endometrioma. These ovarian masses, or "chocolate cysts," can explain the finding of a tender adnexal mass on pelvic examination. The presence of endometriomas is confirmed during laparotomy and may constitute the initial finding for endometriosis. Extrapelvic (e.g. vulva, vagina) or pelvic endometriosis should be considered with any mass or lesion that becomes painful in a cyclic fashion with menses.

Computed tomography (CT) and magnetic resonance imaging (MRI) can provide presumptive evidence of endometriosis but are rarely justified for the initial workup. These expensive imaging studies are not diagnostic, as there is no characteristic appearance or location for endometriosis implants. Pelvic ultrasonography is not diagnostic either but can be helpful in detecting and characterizing pelvic pathology if the pelvic examination is limited owing to tenderness or obesity. Its common availability, lower cost, safety during pregnancy, and nonradiographic nature are general features that prompt many clinicians to include pelvic ultrasonography in the workup of endometriosis.

A cell surface antigen, CA-125, is found on derivatives of coelomic epithelium including endometrial tissue. Despite the correlation of CA-125 levels with both the degree of endometriosis and the response to therapy, the sensitivity of this assay is too low for it to be used as a screening test. The measurement of peritoneal fluid levels appears to be better for detecting minimal and moderate disease. ${ }^{24}$ It may be beneficial to utilize this test when evaluating ovarian cysts. ${ }^{25}$ Developing a serum endometrial antibody assay continues to be investigated, with its sensitivity and specificity greater than 0.9 in detecting endometriosis. ${ }^{25}$

\section{Management $^{26,27}$}

Treatment for endometriosis is preceded by careful confirmation and staging with laparoscopy or laparotomy. Factors to consider prior to treatment include the patient's age and desire for fertility, the severity of symptoms, and the extent, location, and severity of the disease. Even though there is no universally effective cure for this disorder, treatment options do exist that can provide relief of symptoms. The wide variety of treatments for endometriosis betray its poorly defined etiology and pathophysiology. Treatment includes expectant management, conservative surgical therapy, medical therapy, extensive surgery with castration, and superovulation therapies for infertility, such as in vitro fertilization or gamete intrafallopian tube transfer (GIFT). With more advanced endometriosis, there is a greater likelihood that surgical intervention will be required to treat the disease successfully. Surgical removal of both ovaries invariably induces remission of the disease but should be reserved for women with advanced endometriosis who are over 35 years of age and do not intend to become pregnant.

Expectant management, or watchful waiting, may be a reasonable management strategy for the younger woman who desires pregnancy and has mildly symptomatic endometriosis. Efforts can then be spent identifying and correcting any other infertility factors as necessary (see Evaluation of the Infertile Couple, below). This approach has been shown to produce pregnancy rates as high as those achieved with medical therapy or conservative surgery $(50 \%) .^{28}$

Conservative surgery for endometriosis entails either laparoscopy or laparotomy and is indicated (1) for confirmation of diagnosis; (2) if fertility is desired, to determine tubal occlusion or peritubal, pelvic, or ovarian adhesions; (3) for aspiration of chocolate ovarian cysts; and (4) for evaluation of pelvic pain unrelieved by medical therapy. ${ }^{16}$ The initial treatment at the time of diagnostic laparoscopy can often be accomplished with electrocoagulation or laser ablation of implants through the laparoscope. Partial ovarian resection for endometriomas and lysis of adhesions are accomplished as necessary. Definitive surgery includes total hysterectomy and bilateral salpingo-oophorectomy and is indicated in women who do not desire pregnancy and for whom all previous medical and conservative surgical efforts have failed.

Medical management of endometriosis includes the use of danazol, progestins, oral contraceptives, and GnRH agonists. The goal of hormonal therapy is to control symptoms or im- 
prove fertility, or both. Most medical regimens involve treatment for at least 6 months, allowing for adequate regression of implants.

Endometriotic implants behave like normal endometrial tissue and are supported by ovarian hormones. ${ }^{29}$ The effectiveness of hormonal management takes advantage of the biologic response of endometriotic tissue to alterations of the hormonal environment. In either a hypoestrogenic or a hyperandrogenic environment, endometriotic implants become atrophic. Danazol (Danocrine), for instance, induces a hyperandrogenic state, and GnRH agonists produce a hypoestrogenic state. Both agents induce regression of endometriotic implants. Progesterone therapy such as medroxyprogesterone acetate (Cycrin, Provera) induces decidual or atrophic changes in endometriotic implants. Finally, administration of combined estrogen-progestogen contraceptives (pseudopregnancy regimen) produce an acyclic hormonal environment similar to that of pregnancy, which causes endometriotic implants to atrophy. ${ }^{30}$ Table 108.3 summarizes various treatment strategies, the common drugs used for therapy, and their potential side effects. Severe endometriosis and infertility often require an organized treatment plan combining surgical and prolonged medical treatment. In a woman whose symptoms are consistent with endometriosis, empiric medical therapy may be tried prior to definitive surgical diagnosis. In a prospective study it was concluded that after failure of initial treatment with oral contraceptives and NSAIDs, empiric therapy with 3 months of a $\mathrm{GnRH}$ agonist is appropriate. ${ }^{31}$

Infertility associated with endometriosis has now been treated with advanced reproductive techniques including administration of hyperstimulation gonadotropins, in vitro fertilization, and GIFT techniques. ${ }^{32-34}$ The family physician assumes a critical role in managing endometriosis by arranging for appropriate consultation, monitoring medical treatment protocols, and providing long-term emotional support to patients and families troubled by this chronic condition.

\section{Prevention}

At present there are no known effective interventions to prevent endometriosis from developing in a given patient. A positive family history and deferring childbearing lead to a higher likelihood for the development of endometriosis. Endometriosis usually recurs despite medical or conservative surgical treatment. ${ }^{17}$ Long-term hormonal therapy without surgery can help prevent progression in some patients with severe

Table 108.3. Major Treatment Modalities for Endometriosis

\begin{tabular}{|c|c|c|c|c|c|}
\hline Method & $\begin{array}{l}\text { Hormonal } \\
\text { effect }\end{array}$ & Drug & $\begin{array}{c}\text { Route of } \\
\text { administration }\end{array}$ & $\begin{array}{c}\text { Typical } \\
\text { daily dose }\end{array}$ & Problems \\
\hline $\begin{array}{l}\text { Bilateral } \\
\text { oophorectomy } \\
\text { (often with } \\
\text { hysterectomy) }\end{array}$ & Hypoestrogenic & NA & Laparotomy & NA & $\begin{array}{l}\text { Permanent sterility, } \\
\text { bone loss, } \\
\text { menopausal } \\
\text { symptoms }\end{array}$ \\
\hline $\begin{array}{l}\text { Laser or electro- } \\
\text { fulguration } \\
\text { ablation of } \\
\text { endometrial } \\
\text { implants }\end{array}$ & NA & NA & $\begin{array}{c}\text { Laparoscopy or } \\
\text { laparotomy }\end{array}$ & $\mathrm{NA}$ & $\begin{array}{l}\text { Surgical risks; ? } \\
\text { benefit for } \\
\text { severe disease, } \\
\text { recurrence }\end{array}$ \\
\hline \multirow[t]{3}{*}{ GnRH agonist } & Hypoestrogenic & $\begin{array}{l}\text { Nafarelin } \\
\text { (Synarel) }\end{array}$ & Nasal & $\begin{array}{l}0.4-0.8 \mathrm{mg} \text { bid } \\
\times 6 \text { months }\end{array}$ & $\begin{array}{l}\text { Bone loss, hot } \\
\text { flashes, } \\
\text { decreased libido, } \\
\text { vaginal discharge }\end{array}$ \\
\hline & & $\begin{array}{l}\text { Leuprorelin } \\
\text { (Lupron) }\end{array}$ & $\mathrm{IM}$ & $\begin{array}{l}3.75 \mathrm{mg} \text { per month } \\
\text { for } 6 \text { months }\end{array}$ & \\
\hline & & $\begin{array}{l}\text { Goserelin } \\
\quad \text { (Zoladex) }\end{array}$ & SC & $\begin{array}{l}3.6 \mathrm{mg} \text { per month } \\
\text { for } 6 \text { months }\end{array}$ & \\
\hline $\begin{array}{l}\text { Androgenic } \\
\text { agonist }\end{array}$ & Hyperandrogenic & $\begin{array}{l}\text { Danazol } \\
\text { (Danocrine) }\end{array}$ & Oral & $400-800 \mathrm{mg}$ & $\begin{array}{l}\text { Androgenic side } \\
\text { effects, weight } \\
\text { gain, oily skin, } \\
\text { deep voice }\end{array}$ \\
\hline $\begin{array}{l}\text { High } \\
\text { progesterone }\end{array}$ & $\begin{array}{l}\text { Hyperprogestational } \\
\text { (relative low } \\
\text { estrogen) }\end{array}$ & $\begin{array}{l}\text { Medroxyproges- } \\
\text { terone acetate } \\
\text { (Provera), } \\
\text { norethindrone } \\
\text { acetate (Aygestin) }\end{array}$ & Oral & $30-60 \mathrm{mg}$ & $\begin{array}{l}\text { Mood changes, } \\
\text { bloating, } \\
\text { breakthrough } \\
\text { bleeding }\end{array}$ \\
\hline $\begin{array}{l}\text { Continuous oral } \\
\text { contraceptives } \\
\text { (pseudo- } \\
\text { pregnancy) } \\
\text { or cyclic }\end{array}$ & $\begin{array}{l}\text { Combined estrogenic } \\
\text { and progestational }\end{array}$ & $\begin{array}{l}\text { Birth control pills } \\
\text { (many types) }\end{array}$ & Oral & $\begin{array}{l}\text { e.g., } 0.035 \\
\text { mg ethinyl- } \\
\text { estradiol } / 1 \mathrm{mg} \\
\text { norethindrone } \\
\text { (1-4 per day) }\end{array}$ & $\begin{array}{l}\text { Oral contraceptive } \\
\text { side effects: } \\
\text { mood changes, } \\
\text { weight gain, } \\
\text { bloating, } \\
\text { hypertension }\end{array}$ \\
\hline
\end{tabular}

$\mathrm{NA}=$ not available; $\mathrm{SC}=$ subcutaneous. 
symptoms. Only definitive surgery such as abdominal hysterectomy with bilateral salpingo-oophorectomy, combined with resection of all endometrial implants, yields the highest likelihood for resolution of symptoms. The laparoscopic finding of minimal endometrial disease in a woman not desiring pregnancy can frequently be managed with cyclic birth control pills to lessen further seeding. More advanced disease usually requires 6 months of danazol or medroxyprogesterone acetate followed by cyclic birth control pills. ${ }^{20}$

\section{Family and Community Issues}

Education regarding endometriosis ideally begins during early adolescence as part of comprehensive health education. Women should be encouraged to seek medical help for the symptoms of endometriosis. For many women endometriosis becomes a chronic affliction that requires ongoing education, treatment, and compassion. If the patient requests, the physician should meet with other members of the family to offer information and address questions or concerns. Patients and families may benefit from contacting the Endometriosis Association to obtain educational materials and information regarding support groups that deal with the impact of this chronic condition. ${ }^{35}$ Women with documented endometriosis who desire pregnancy should be counseled regarding its relation to infertility, so these desires and the timing of child bearing can be discussed. Effectively managing patients with endometriosis challenges the family physician to remain an informed patient advocate, as comprehensive care frequently involves referral and familiarity with long-term treatment modalities not routinely used by family physicians. ${ }^{36}$

\section{Evaluation of the Infertile Couple}

Infertility is generally diagnosed when pregnancy has not occurred in a couple trying to conceive after 1 year of unprotected intercourse. It is estimated that nearly $15 \%$ of couples in the United States are infertile. ${ }^{37}$ The infertility risk doubles for women of ages 35 to 44 compared to women of ages 30 to 34 ; consequently, about one third of women older than 35 who desire pregnancy experience infertility. ${ }^{38}$

Couples are seeking professional help for infertility on a increasing basis, and family physicians are in an ideal position to begin this evaluation. Their familiarity with partner's family, medical, and physical histories allow the assessment to begin with both partners, which contributes to the cost-effectiveness of the family physician's infertility evaluation. The major goals when evaluating the infertile couple include (1) identifying and correcting causes of infertility, (2) providing accurate information for the couple, (3) providing emotional support during and after the evaluation process, and (4) providing counseling regarding alternatives should pregnancy be unlikely or impossible. ${ }^{39}$ This discussion presents the initial workup of the infertile couple, which is sufficient to allow diagnosis in approximately $60 \%$ of couples seeking help. ${ }^{40}$

There are various estimates regarding the causes of infertility. Table 108.4 summarizes the experience for infertile couples in the United States. ${ }^{39-41}$

\section{Table 108.4. Male and Female Infertility Factors}

\author{
Male factors ( $40 \%$ of couples) \\ Disorders of spermatogenesis \\ Obstruction of efferent ducts \\ Disorders of sperm motility \\ Sexual dysfunction \\ Female factors ( $45 \%$ of couples) \\ Pelvic origin ( $30-50 \%$ of female causes) \\ Tubal disorders \\ Uterine disorders \\ Endometriosis \\ Ovulatory origin ( $40 \%$ of female causes) \\ Endocrine (adrenal, thyroid, pituitary) disorder \\ Ovarian: luteal phase defects \\ Cervical origin ( $10 \%$ of female causes) \\ Mucous problems \\ Infectious problems \\ Combined factors (15-30\% of couples) \\ Unidentifiable factors ( $15 \%$ of couples)
}

\section{Initial Fertility Workup}

A timely and cost-effective approach to evaluating the infertile couple requires initial evaluation of both the male and female factors for infertility. Table 108.5 summarizes an organized approach to the fertility workup. ${ }^{39-42}$ Both partners are scheduled for extended examination times. Ample time is afforded to explain the need and methods for the various initial tests and data collection including (1) semen analysis, (2) documentation of ovulation, (3) postcoital test, and (4) evaluation of tubal patency. The exact sequence in which a physician evaluates a couple's infertility varies based on historical and physical findings ascertained during the initial visit.

A semen sample is analyzed within 2 hours of ejaculation after 48 hours of ejaculatory abstinence. Evaluation should be by persons experienced with fertility semen analysis. If an abnormality is found on this initial assessment, two additional semen analyses are performed 2 weeks apart. If the abnormality persists, urologic consultation is appropriate. ${ }^{43}$

Assessing ovulation can be accomplished by one or more methods: (1) basal body temperature (BBT) assessment; (2) timed serum progesterone levels; (3) urinary luteinizing hormone (LH) screening; and (4) endometrial biopsy. The method of recording BBT data is explained during the initial visit. Serum progesterone levels are measured 7 days after estimated ovulation. Values consistent with ovulation vary with each laboratory, but generally values of more than $15 \mathrm{ng} / \mathrm{mL}$ are consistent with normal ovulatory function and levels under $5 \mathrm{ng} / \mathrm{mL}$ imply anovulation.

Endometrial biopsy, in addition to histologically detecting ovulation, provides timing of the progestational effect with the day of the cycle and can provide information regarding ovulation and luteal phase defects. Endometrial sampling is easily accomplished using small aspiration catheters (Pipelle, Z-sampler) at as close to 7 days after presumed ovulation as possible (roughly, day 22 of a 28 -day cycle).

Postcoital testing requires evaluation of the sperm-mucus interaction. The couple is instructed to have intercourse following 48 hours of abstinence. Pelvic examination is per- 


\section{Table 108.5. Evaluation Plan for the Infertile Couple}

Initial visit (extended visit time for both partners)

Complete medical, family, drug and sexual history

Laboratory studies

Sexually transmitted disease cultures (especially Chlamydia, Ureaplasma urealyticum, Neisseria gonorrhoeae)

Assess need for HIV screening

Consider CBC, FBS, VDRL, urinalysis

Men: semen analysis

Women

Papanicolaou screening

$\mathrm{KOH}$ and wet mount

Focused studies

Thyroid history? - thyroid function tests

Galactorrhea?-prolactin levels

Abnormal pelvic examination?-ultrasonography, laparoscopy

History PID? - hysterosalpingography

Endometriosis likely? - laparoscopy

History sexual dysfunction?-education, counseling

Ovulatory monitoring (BBT, urinary $\mathrm{LH}$

Education

Alcohol, drugs, hot tubs, sexual practices/frequency, douching, lubricants

Arrange for postcoital sample analysis with next visit

Second visit (midcycle/ovulatory as predicted by BBT and/or urinary LH testing)

Review laboratory studies, semen analysis, ovulatory monitoring data to date

Analyze postcoital sample

Consider and discuss need for endometrial biopsy

Arrange for hysterosalpingography during follicular phase

(days 7-9); if laparoscopy deemed necessary, HSP often accomplished during laparoscopy

Third visit (midluteal phase of cycle, 7-9 days postovulation) Review studies to date

Perform endometrial sampling

Consider progesterone and prolactin levels if not already done

Consider HSP or laparotomy; referral often appropriate at this time

$\mathrm{CBC}=$ complete blood counts; $\mathrm{FBS}=$ fasting blood sugar $\mathrm{LH}=$ luteinizing hormone; $\mathrm{HSP}=$ hysterosalpingography; $\mathrm{BBT}=$ basal body temperature; HIV = human immunodefi ciency virus; PID = pelvic inflammatory disease.

formed and aspirated cervical mucus is examined within 2 to 8 hours of intercourse. Intercourse should occur within 24 to 48 hours of presumed ovulation as determined by methods outlined above. The postcoital mucus is examined by experienced individuals for quantity, clarity, $\mathrm{pH}$, spinnbarkeit (ability of cervical mucus to stretch), and the number, forms, and motility of sperm. Generally, finding 5 to 10 sperm with linear motility per high-power field in clear, acellular mucus with more than $8 \mathrm{~cm}$ spinnbarkeit excludes cervical factors as a major cause of infertility.

During a subsequent office visit, results of the initial laboratory studies, cultures, and semen analysis are discussed. The postcoital sample can be obtained, and an appointment for the endometrial biopsy, luteal progesterone, and prolactin levels scheduled. The results of these studies are carefully reviewed during the third visit, at which time a decision is made to pursue hysterosalpingography, laparoscopy, hysteroscopy, or laparotomy. The frequency of these visits also facilitates emotional support and encouragement for the couple. RESOLVE (5 Water Street, Arlington, MA 02174), a national nonprofit infertility organization, has chapters throughout the United States and is an excellent resource for support and counseling groups. ${ }^{42}$

\section{Further Evaluation}

Hysterosalpingography (HSP) is a safe, high-yield procedure when performed during the early proliferative phase of the cycle. During HSP a special catheter is passed through the vagina into the endocervical canal through which contrast medium is injected and fluoroscopically followed through the endometrial and fallopian tube lumen. An undiagnosed pelvic mass or PID contraindicates this procedure, as does an iodine or radiocontrast dye allergy. HSP can be performed during laparoscopic surgery as well. The appearance of uterine defects on HSP implicates a uterine cause of infertility, which include post-diethylstilbestrol (DES) uterine abnormalities ( $T$-shaped, hypoplastic cavity), intrauterine synechiae (Asherman syndrome), submucous or large intramural myomas, congenital anomalies, and leiomyomas.

Further evaluation of pelvic factors for infertility requires laparoscopy. Laparoscopy is indicated if HSP is contraindicated or abnormal and is also performed if historical (e.g., PID, endometriosis) or physical (e.g., adhesions, tube/ovarian abnormality) findings implicate a pelvic cause. Women with endometriosis are twice as likely to be infertile as women without this condition. Endometriosis is confirmed and staged by laparoscopy. Treatment for endometriosis was discussed earlier in this chapter. Finally, laparoscopy is indicated if no other significant cause of infertility is identified.

Ovulatory causes of infertility include adrenal and thyroid disorders. Fasting blood glucose, thyroid function, folliclestimulating hormone (FSH), luteinizing hormone (LH), and prolactin levels assist the evaluation of endocrinologic factors for infertility. Drug-related infertility must also be considered, as a number of medications contribute to disruption of thyroid, adrenal, or ovarian functioning in women and thyroid or adrenal functioning in men (e.g., antihypertensive drugs, major tranquilizers, steroids). Therapy for ovulatory factors is directed toward the underlying disorder, including thyroid replacement for hypothyroidism, bromocriptine for hyperprolactinemia (with pituitary macroadenoma ruled out), or clomiphene to induce ovulation.

Cervical factors for infertility are assessed initially by the postcoital test. The finding of leukocytes suggests cervicitis, and cultures for gonorrhea, Ureaplasma urealyticum, and Chlamydia are useful for directing treatment. Finding nonmotile or nonprogressively motile sperm with a "shaking" pattern suggests sperm antibodies. Miscalculation of ovulation timing is suggested by poor-quality cervical mucus, which is abnormally thick, cloudy, and demonstrates poor spinnbarkeit. A repeat postcoital test is necessary if ovulatory timing is in question.

Despite comprehensive evaluation $5 \%$ to $10 \%$ of couples have no identifiable cause for their infertility. Often referral to infertility centers for consideration of assisted reproductive 
technologies (ARTs) is necessary (e.g., in vitro fertilization, GIFT) and appropriate. Attitudes regarding adoption and assisted reproductive technologies should be explored.

Often individual or joint guilt regarding infertility can be underestimated. Family physicians are in an ideal position to utilize established trust and comfort with their patients to explore potential past history and guilt issues that might relate to infertility. Emotional support remains a part of every visit. ${ }^{44}$

\section{Toxic Shock Syndrome}

In 1978 an acute, febrile, exanthematous illness associated with multisystem organ failure became known as the toxic shock syndrome (TSS). ${ }^{45}$ There are currently no definitive tests or specific serologic markers for diagnosing TSS. The diagnosis requires the presence of the six clinical criteria, outlined in Table 108.6. ${ }^{46,47}$ TSS should be suspected in all patients who present with flu-like symptoms, rash, and otherwise unexplained hypotension.

Early reports indicate that more than $90 \%$ of the reported TSS cases involved menstruating females. ${ }^{48}$ Because of the high correlation of TSS with menstruation and tampon use, the perception of TSS as strictly a "tampon disease" persists. More recent data reveal that only half of the reported cases were female, and many of these cases were not associated with menstruation. ${ }^{49}$ Most cases of tampon-related TSS occurred during the teenage years; $97 \%$ of reported patients were white, and $42 \%$ were adolescents. ${ }^{50}$ Physicians must recognize that TSS can occur in nonmenstruating women, men, and children of all ages. Nonmenstrual cases of TSS occur in males and females equally with various sites and types of infection, including surgical wounds, burns, abscesses, sinus infections, bronchopneumonia, influenza $\mathrm{A}$, tracheitis, empyema, septic abortion, intravenous drug injections, pilonidal abscess, nasal packing, insect or human bites, diaphragm use, and even ear piercing. Interestingly, menstruation-related TSS affects Caucasians almost exclusively, whereas non-menstruationrelated forms affect the races proportionate to the racial $/ \mathrm{sex}$ ual mix of the population. ${ }^{51,52}$

\section{Pathogenesis}

The expression of TSS depends on the interaction of several bacterial and host factors that are incompletely understood

Table 108.6. Toxic Shock Syndrome, Criteria for Diagnosis Confirmed diagnosis requires that all six criteria be met:

1. Acute fever

2. Scarlatiniform rash

3. Desquamation of palms and soles $1-2$ weeks after illness onset

4. Hypotension

5. Clinical or laboratory evidence of involvement of at least three organ systems: hematologic, gastrointestinal, neurologic, cardiovascular, hepatic, renal, muscular

6. Other causes excluded (e.g., sepsis, measles, drug or toxic ingestion)

Source: Data from Todd and Fishaut ${ }^{45}$ and Tofte and Williams. ${ }^{46}$
In the original studies, patients with TSS usually had a source of staphylococcal infection. More recent reports have characterized a streptococcal toxic shock-like syndrome caused by toxins elaborated by group A streptococci. ${ }^{53,54}$ Both have in common the presence of bacteria-related toxins that appear to initiate the serious multiorgan syndrome characteristic of these disorders. Toxic shock syndrome toxin-1 (TSST-1) has been identified as one of the significant staphylococcal mediators of pathogenicity in TSS, ${ }^{55}$ although TSS can occur in the absence of TSST-1. Endotoxin from co-infecting gramnegative or streptococcal bacteria has also been implicated in TSS. ${ }^{56}$ Staphylococcal toxins may interact synergistically with these endotoxins. TSST-1 enhances the release of a wide variety of endogenous mediators produced by the host, which partly explains the multiorgan dysfunction characteristic of this syndrome. Finally, the correlation of TSS with vaginal materials such as tampons, barrier sponges, or the diaphragm strongly implies an as yet uncharacterized relation between foreign materials and the induction and maintenance of this condition. TSS apparently results from multiple, cumulative effects of primary and secondary mediators initiated by the continued growth of certain bacteria.

\section{Clinical Presentation}

Toxic shock syndrome presents with a broad spectrum of clinical findings ranging from relatively mild symptomatology to a rapidly fatal illness. ${ }^{56,57}$ Mortality is about $3 \%$. Patients may progress from onset of symptoms to overt multisystem failure within 48 hours. Hypotension and poor tissue perfusion, with nonhydrostatic leakage of fluid from the intravascular to the interstitial space, accounts for a portion of the multisystem organ failure (renal, hepatic, central nervous system, hematologic). Fatal complications include refractory shock, renal failure, arrhythmias, intravascular coagulopathy, and respiratory distress syndrome. The initial fever, rash, and malaise of TSS may mimic a common viral syndrome early in the disease process. These symptoms typically progress rapidly to include high fever $\left(104^{\circ} \mathrm{F}\right)$, pharyngitis, conjunctivitis, diarrhea, vomiting, myalgia, and scarlet fever-like rash. Within a matter of hours orthostatic dizziness, fainting, or overt hypotension may occur; these first symptoms clearly set this syndrome apart from benign infectious processes. The rash of TSS is usually a prominent finding and typically appears as a diffuse scarlatiniform exanthem beginning on the trunk and spreading to the arms and legs with flexural accentuation. Some clinicians have described this rash as having a "sunburn" appearance that blanches with pressure. "Strawberry tongue," as seen with scarlet fever, is a common finding. Erythema of the mucous membranes and intense conjunctival hyperemia without purulence are characteristic. Generalized nonpitting edema accompanies the rash in many cases. Desquamation of the hands and feet is commonly seen within 10 to 21 days after presentation. Reversible patchy alopecia and shedding of fingernails has been described as well. $^{58}$

Toxic shock syndrome is rare in children, but when it does appear the clinical features of the illness do not differ appreciably from those described in adults. There does seem to be a 
higher likelihood of respiratory involvement in children. ${ }^{55}$ The differential diagnosis of TSS in children includes Kawasaki disease, staphylococcal scalded skin syndrome, scarlet fever, Rocky Mountain spotted fever, leptospirosis, erythema multiforme, Stevens-Johnson syndrome, and measles.

Common laboratory findings of TSS reflect widespread organ involvement: sterile pyuria; normocytic anemia; leukocytosis with left shift; prolongation of the prothrombin and partial thromboplastin times; an increase in serum bilirubin, creatinine, and creatine kinase; hyponatremia; hypokalemia; and metabolic acidosis. ${ }^{59}$

\section{Management}

Hospitalization is necessary for all patients with presumed TSS, as the illness may progress from mild to life-threatening within a matter of hours. Supportive measures tailored to the patient's symptoms are the mainstay of therapy. ${ }^{60} \mathrm{~A}$ search is made for the potential source of the staphylococcal or streptococcal toxin, and cultures of any suspected source are obtained. Removal of vaginal tampons, pads, sponges, or diaphragms is paramount. Recent surgical wounds are explored even if they appear not to be inflamed or infected. All presumed sources of toxin must be thoroughly drained and irrigated.

Prospective management includes administration of large volumes of colloid and crystalloid fluids with glucose (10-20 $\mathrm{mL} / \mathrm{kg}$ ) over the first hour to reestablish vascular volume. Patients may require 5 to $10 \mathrm{~L}$ of fluid during the first 24 hours to maintain tissue perfusion and urinary output. Swan-Ganz catheterization is required for severe cases. After specimens for culture are obtained, antistaphylococcal antibiotics (e.g., methicillin, oxacillin, nafcillin, first-generation cephalosporin, or vancomycin) are given intravenously until oral fluids can be tolerated. Antibiotics are continued for at least 10 days. Steroid therapy (e.g., methylprednisolone $10-30 \mathrm{mg} / \mathrm{kg} / \mathrm{day}$ ) may be given to patients with severe unresponsive shock. Intravenous immune globulin may be also given to patients who are not improving, although the effectiveness of this therapy has not been proved.

Signs of adult respiratory distress syndrome (ARDS) often develop on the second or third day of treatment with severe TSS. Intubation with continuous positive airway pressure may be required; generally, fluids are not restricted. Acute renal failure may require dialysis.

Recurrences of TSS are common but generally milder. Female patients who experience TSS are advised to avoid the use of vaginal devices (tampons, sponges, diaphragms). Prophylactic antistaphylococcal oral antibiotics at the time of menses have been recommended for patients experiencing TSS associated with menses. Oral contraceptives have been shown to decrease the risk of TSS recurrence. ${ }^{48}$ These approaches have not yet been conclusively supported by rigorous studies.

\section{References}

1. Peterson HB, Hulka JF, Phillips JM. American Association of Gynecologic Laparoscopists' 1988 membership survey on operative laparoscopy. J Reprod Med 1990;35:587-9.
2. Nolan TE, Elkins TE. Chronic pelvic pain: differentiating anatomic from functional causes. Postgrad Med 1993;94:125-8.

3. Toomey TC, Hernandez JT, Gittlelman DF, Hulka JF. Relationship of sexual and physical abuse to pain and psychological assessment variables in chronic pelvic pain. Pain 1993;94: 125-8.

4. Ling FW, Slocumb JC. Use of trigger point injections in chronic pelvic pain. Obstet Gynecol Clin North Am 1993;20:809-15.

5. King Baker P. Musculoskeletal origins of chronic pelvic pain. Obstet Gynecol Clin North Am 1993;20:719-40.

6. King PM, Myers LA, Ling FW, et al. Musculoskeletal factors in chronic pelvic pain. J Psych Obstet Gynaecol 1991;12:87-98.

7. Basu HK. Major common problems: pelvic pain. Br J Hosp Med 1981;26:150-8.

8. Vercellini P, Fedele L, Arcaini L, et al. Laparoscopy in the diagnoses of chronic pelvic pain in adolescent women. J Reprod Med 1989;34:827-32.

9. Baker PN, Symonds EM. The resolution of chronic pelvic pain. Am J Obstet Gynecol 1992;166:835-9.

10. McQuay HJ, Carroll D, Glynn CJ. Low dose amitriptyline in the treatment of chronic pain. Anesthesia 1992;47:646-52.

11. Parsons L, Stovall T. Surgical management of chronic pelvic pain. Obstet Gynecol Clin North Am 1993;20:765-78.

12. Stovall TG, Ling FW, Crawford DA. Hysterectomy for chronic pelvic pain of presumed uterine etiology. Obstet Gynecol 1990; 75:676-81

13. Sampson JA. Perforating hemorrhagic (chocolate) cysts of the ovary, their importance and especially their relation to pelvic adenomas of the endometrial type. Arch Surg 1921;3:245-323.

14. Saltiel E, Garabedian-Ruffalo SM. Pharmacologic managemen of endometriosis. Clin Pharm 1991;10:518-30.

15. Rawsn JM. Prevalence of endometriosis in asymptomatic women. J Reprod Med 1991;36:513-5.

16. Dawood MY. Endometriosis. In: Gold JJ, Josimovich JB, eds. Gynecologic endocrinology. New York: Plenum, 1987;387-404

17. Moghissi KS. Office management of endometriosis. In Stenchever MA, ed. Office gynecology. St. Louis: Mosby-Year Book, 1992;413-29.

18. National Center for Health Statistics, McCarthey E. Inpatient utilization of short-stay hospitals by diagnosis: United States 1980. Hyattsville, MD: National Center for Health Statistics, 1982. DHSS Publ. no. (PHS) 83-1735. (Vital and health statistics; series 13: Data from the National Health Survey, no. 74.)

19. Hurst BS, Rock JA. Endometriosis: pathophysiology, diagnosis, and treatment. Obstet Gynecol Surv 1989;44:297-304

20. Endometriosis and infertility. In: Speroff L, Glass RH, Kase NG, eds. Clinical gynecology, endocrinology, and infertility, 4th ed. Baltimore: Williams \& Wilkins, 1989;547-63.

21. Dmowski WP. Endometriosis. In: Glass RH, ed. Office gynecology. Baltimore: Williams \& Wilkins; 1987;317-36.

22. Berube S, Marcoux S. Langeuiun M, Maheux R. Fecundity of infertile women with minimal or mild endometriosis and women with unexplained infertility. Canadian Collaborative Group of Endometriosis. Fertil Steril 1998;69:1034-41

23. American College of Obstetricians and Gynecologists. Management of endometriosis. Technical bulletin no. 85. Washington, DC: ACOG, 1985.

24. Colacurci N, Fortunato N, DeFranciscis P, et al. Serum and peritoneal CA-125 levels as diagnostic test for endometriosis. Eur J Obstet Gynecol Repord Biol 1996;66:41-3.

25. Mathur S. Autoimmunity in endometriosis: relevance to infertility. Am J Reprod Immunol 2000;44:89-95.

26. Koninck PR, Riittinen L, Sepalla M, Cornillie FJ. CA-125 and placental protein 14 concentrations in plasma and peritoneal fluid of women with deeply infiltrating pelvic endometriosis. Fertil Steril 1992:57:523-30.

27. Badawy SZA, Cuenea V, Freliech H, Stefanu C. Endometria 
antibodies in serum and peritoneal fluid of infertile patients with and without endometriosis. Fertil Steril 1990;53:930-2.

28. Seibel MM. Does minimal endometriosis always require treatment? Contemp Obstet Gynecol 1989:34:27-39.

29. DiZerega GS, Barber DL, Hodgen GD. Endometriosis: role of ovarian steroids in initiation, maintenance, and suppression. Fertil Steril 1980;33:649-53.

30. Barbieri RL. Endometriosis 1990: current treatment approaches. Drugs 1990;39:502-10.

31. Ling FW. Randomized controlled trial of depot leuprolide in patients with chronic pelvic pain and clinically suspected endometriosis. Pelvic Pain Study Group. Obstet Gynecol 1999;93 51-8.

32. Fedele L, Bianchi S, Marchin M, Villa L, Brioschi D, Parazzin $\mathrm{F}$. Superovulation with human menopausal gonadotropins in the treatment of infertility associated with minimal or mild endometriosis: a controlled randomized study. Fertil Steril 1992;58:28-31.

33. Medical Research International Society for Assisted Reproductive Technology, American Fertility Society. In vitro fertilization-embryo transfer (IVF-ET) in the United States: 1989 results from the IVF-ET registry. Fertil Steril 1991;55:14-23.

34. Olive DL, Schwartz BL. Endometriosis. N Engl J Med 1993 328:1759-69.

35. Overcoming endometriosis: new help from the Endometriosis Association. Milwaukee: Endometriosis Association.

36. American College of Obstetrics and Gynecologists. Important facts about endometriosis. Washington, DC: ACOG.

37. Whitman-Elia GF, Baxley EG. A primary care approach to the infertile couple. J Am Board Fam Pract 2001;14(1):33-45.

38. Mosher WD. Infertility trends among U.S. couples: 1965-1976. Fam Plann Perspect 1982;14:22-7.

39. Speroff L, Glass RH, Kase NG, eds. Female infertility, 6th ed Baltimore: Williams \& Wilkins, 1999;809-39.

40. Zarutskie PW. Evaluation of the infertile couple. In: Stencheve MA, ed. Office gynecology. St. Louis: Mosby Year Book, 1992; $441-70$

41. American College of Obstetricians and Gynecologists. Infertility. Technical bulletin no. 125. Washington, DC: ACOG, 1989.

42. Davajan V. Workup of the infertile couple. In: Mishell DR, Brenner PF, eds. Management of common problems in obstetrics and gynecology. Oradell, NJ: Medical Economics Books. $1984 ; 387-93$.
43. Kolettis PN, Sabanegh ES. Significant medical pathology discovered during a male infertility evaluation. J Urol 2001;166(1): 178-80.

44. Boivin J, Appleton TC, Baetens P, et al. Guidelines for counseling in infertility: outline version. Hum Reprod 2001;16(6): $1301-4$.

45. Todd J, Fishaut M. Toxic-shock syndrome associated with phage-group-I staphylococci. Lancet 1978;2:1116-8.

46. Tofte RW, Williams DN. Toxic shock syndrome: evidence of a broad clinical spectrum. JAMA 1981;246:2163-7.

47. Bryner CL Jr. Recurrent toxic shock syndrome. Am Fam Physician 1989:39:157-64

48. Davis JP, Chesney PJ, Wand PJ, LaVenture M. Toxic shock syndrome: epidemiologic features, recurrence, risk factors, and prevention. N Engl J Med 1980;303:1429-35.

49. Centers for Disease Control. Summary of notifiable disease, United States. MMWR 1988;37:40.

50. Litt IF. Toxic shock syndrome-an adolescent disease. J Adolesc Health Care 1983;4:270-4.

51. Reingold AL, Hargrett NT, Dan BB, Shands KN, Strickland BY, Broome CV. Nonmenstrual toxic shock syndrome: a review of 130 cases. Ann Intern Med 1982;96:871-4.

52. Sion ML, Hatzitolios AI, Toulis EN, Mikoudi KD, Ziakas GN Toxic shock syndrome complicating influenza $\mathrm{A}$ infection: a two-case report with one case of bacteremia and endocarditis. Intensive Care Med 2001;27(2):443-8.

53. Gallo UE, Fontanarosa PB. Toxic streptococcal syndrome. Ann Intern Med 1990;19:1332-4.

54. Wolf J, Rabinowitz L. Streptococcal toxic shock like syndrome. Arch Dermatol 1995;131:73-7.

55. Resnick SD. Toxic shock syndrome: recent developments in pathogenesis. J Pediatr 1990;116:321-8.

56. Parsonnet J. Mediators in the pathogenesis of toxic shock syndrome: overview. Rev Infect Dis 1989;2(suppl 1):s263-9.

57. Herzer CM. Toxic shock syndrome: broadening the differential diagnosis. J Am Board Fam Pract 2001;14(2):131-6.

58. Tofte R, Williams DN. Toxic shock syndrome: clinical and laboratory features in 15 patients. Ann Intern Med 1981:94:149-56.

59. Davis JP, Osterholm MT, Helms CM, et al. Tri-state toxic-shock syndrome study: clinical and laboratory findings. J Infect Dis 1982;145:441-8

60. Todd JK. Therapy of toxic shock syndrome. Drugs 1990;39: 856-61. 